\title{
TRUST BUILDING FOR SMES THROUGH AN E-ENGINEERING HUB
}

\author{
Z. Ren, T. M. Hassan and C.D. Cater \\ Dept. of Civil \& Building Engineering, Loughborough University, UK, z.ren@lboro.ac.uk
}

\begin{abstract}
Trust building is a vital issue and enabler for e-Business. It has been studied at various stages of e-Business from initial trust establishment, contract negotiation and formation, fulfilment to closure. Current studies mainly focus on building trust through the improvement of the social business environment and online security by adopting latest technologies. This paper introduces a new trust building approach developed in the eHUBs (e-Engineering enabled by Holonomic and Universal Broker Services) project. Unlike most of the current trust building studies, this approach fosters trust building between Small and Medium sized Enterprises (SMEs) and their clients through collaborative project planning (PP). The e-Hub provides a transparent, traceable and predictable collaborative PP and contract negotiation platform.
\end{abstract}

\section{INTRODUCTION}

Small and Medium sized Enterprises (SMEs) are considered the backbone of the European economy, as well as an important key source of jobs and a breeding ground for the knowledge-based economy. By focusing on their core competencies and knowledge, the flexible integration of resources and engineering collaboration of SMEs is expected to facilitate the strengthening of the competitive position of SMEs in their struggle for the future. Recent surveys (e-HUBs Technical Annex, 2001) also show large companies are willing to consider the outsourcing of non-core competency tasks to specialized SMEs. Rather than adopting the "extended enterprise" formula, SMEs are often involved in 'on-the-fly' partnerships. Efficient integration of engineering services on an ad-hoc basis into engineering projects is of strategic importance for SMEs. The knowledge intensive businesses that SMEs are often involved in are characterized by flexibility, decentralization and networking of organizations. These factors have led SMEs to look for platforms to initiate partnerships that are remote, time-critical and volatile.

The emergence of B2B e-Markets is expected to provide such platforms. By taking advantage of e-Markets' services, SMEs are able to conduct procurement and collaboration online, and therefore reach a wider market than ever before. However, despite the assurances of technological security mechanisms, there appears to be a perception that business through e-Markets may be both insecure and unreliable due to the uncertainties inherent in the e-Business environment, particularly for SMEs as they are more vulnerable to external threats than their larger counterparts. Risks exist not only due to the ad-hoc and volatile pattern of business, but also due to 
SMEs' unfamiliarity with the technology and not commonly, a shortage of resources.

Although considerable research on trust in e-Business is ongoing, much of it concentrates on trust building in $\mathrm{B} 2 \mathrm{C}$ environments; very few studies have been undertaken on trust in the context of e-collaboration for SMEs in dynamic business environments. Moreover, current trust building studies in e-Business focus either on technology aspects (e.g. improvement of online payment security, information access and authority) or social aspects (e.g. involvement of trust third party, selfregulations and legal systems). No study has been done on trust building through collaborative engineering activities (e.g. collaborative project preparation) or eEngineering platforms (e.g. increasing the transparency of e-Engineering market), which has been identified as a key trust contributor in engineering procurements and collaborations (Elo, URL1; Andersson and Sörvik, URL2; Ong, URL3; EC, 1997).

This study developed a novel approach, the e-Engineering Hub, to facilitating trust building between Engineering Service Providers (ESPs) and their clients through collaborative project preparation and planning performed on the e-Hub workspace. The e-Hub provides a transparent, traceable and predicable collaborative workspace. By conducting collaborative project planning (PP) on the e-Hub, both partners can clearly understand what to do, how to do it, and when to do it. This provides a chance for project partners to understand each other more clearly. This is particularly important for SMEs to demonstrate their expertise and competence to their partners. Trust is fostered through the effective collaborative PP.

This paper first reviews the trust concept, key trust building issues, and trust building approaches in e-Business; then introduces the e-Hub concept and presents the functional architecture of the e-Engineering Hub. It then discusses the trust building process in a seismic engineering testbed with focus on the functional architecture. Finally, conclusions are drawn based on the result of the study.

\section{TRUST AND TRSUT BUIDLING}

Trust is seen as the coordinating mechanism which binds relationship together, provides the necessary flexibility, lowers transaction costs and reduces the complexity of relationships. Particularly, trust is a key determining factor for commercial relationships wherever risk, uncertainty, or interdependence exist (McKnight and Chervany, 2001). Given its importance, trust has become a major concern in the e-Business environment over the past decade.

\subsection{Trust Definition}

Due to the different research perspectives and constructs adopted to conceptualize trust, trust has been defined in literally dozens of ways. Inconsistent and incomplete conceptualization leads to problems in the development, operation and measurement of trust. In an e-Engineering Hub context, two kinds of trust definitions have been adopted as below. The first type is used to define users' trust to the e-Engineering $\mathrm{Hub}$ whilst the second type is used to define the trust among business partners. 
- Blau (1964): "The belief that a party's word or promise is reliable and that a party will fulfil his obligations in an exchange relationship".

- Mayer et al. (1995): "The willingness of a party to be vulnerable to the actions of another party based on the expectation that the other will perform a particular action important to the trustor, irrespective of the ability to monitor or control the other party".

\subsection{Trust Building Issues}

After clarifying the trust definition, this section further explores the nature and characteristics of trust, key trust building issues and the trust development process.

1) Schumacher (URL4) summarised that most conceptualisations of trust identify several main factors:

- Firstly, trust exists in an environment of uncertainty and risk. If parties could undertake a transaction with complete certainty, then trust would not be required and the concept would be trivial;

- Secondly, trust implies the vulnerability of a partner. The extent of the potential loss due to untrustworthy behaviour is typically much greater than the anticipated gains from honest actions;

- Thirdly, trust describes some degree of predictability. It reflects a prediction of a party's behaviour, and implies that the expectancy that it will perform a specific action is high enough for another party to consider engaging in interaction;

- Fourthly, trust exists in an environment of interdependence and mutuality. The parties to an exchange have to believe that their own objectives cannot be realised without reliance upon the other; and

- Fifthly, trust is inherently a positive and good notion. When people refer to trust, they are making a statement about the likelihood of positive outcomes.

2) Blomqvist and Ståhle (URL5) summarised that competence, goodwill, behaviour and self-reference are four key components of trust in technology partnerships. Trust is increased by, and decreased by the lack of, evidence of these components in partners' actual behaviour and communication.

- Competence represents a party's technological capabilities, skills, expertise and know-how. It is a necessary antecedent for trust in the business context, especially in technology partnerships, where complementary technological knowledge and competencies are a key motivation for partnership formation.

- Goodwill implies a partner's moral responsibility and positive intentions towards the other. Expected goodwill is a necessary and active component for trust in any business partnership formation.

- When the relationship is developing, the actual behaviour (e.g. that the trustee fulfils the positive intentions) enhances trustworthiness. The capability and goodwill dimensions of trust become visible in the behavioural signals of trustworthiness.

- Self-reference means a system's capability of autonomy and dependency. Selfreference is demonstrated by the system's ability to define its own existence, the basic idea for being and doing, values, principles and goals, as well as abilities.

3) McKnight and Chervany (1996) provided a typology of interrelated types of trust constructs that helps to distinguish and capture the conceptual meanings of trust and the key trust construct factors. They identified three major trust types: dispositional, 
institutional, and trusting beliefs: Dispositional trust, Institutional trust and Trust beliefs.

Trusting beliefs means that one believes that the other party has one or more characteristics beneficial to oneself. It reflects the idea that interactions between people, and cognitive-emotional reactions to such interactions, determine behaviour. There are four types of trusting beliefs: competence, benevolence (i.e. believes that the other party cares about one and is motivated to act in one's interest.), integrity (i.e. believes that the other party makes good-faith agreements, tells the truth, acts ethically, and fulfils promises.) and predictability.

These studies point out the key components and conditions for trust building from various aspects. It is always a hard topic to identify or demonstrate these factors so that trust, especially for SMEs, can be built in e-Business / e-Engineering.

\subsection{Trust Building Approaches in e-Business}

Compared with trust building in general business, trust in e-Business has at least three different perspectives: trading partner trust as between organisations in eBusiness (e.g. psychological or behaviour trust, interpersonal/organisational trust and product trust); technology assurances (e.g. security-based mechanisms provided by e-Business platform); and external legal situations (e.g. legal systems and selfregulation). To attract more business opportunities, e-Market providers have taken both business approaches and technology approaches to overcome trust barriers. For example, some of the business approaches include:

- Providing unconditional guarantees of safety with an offer to cover any losses due to credit card fraud (e.g. Amazon);

- Providing detailed explanations of privacy policies on their Websites (e.g. Travelocity);

- Capitalizing on existing brand reputations in the case of established businesses (e.g. Microsoft Expedia, Barnes and Noble);

- Including suppliers only approved by Trusted Third Parties (TTP), trust seals, trust signals or intermediaries (e.g. Consumer Reports, Trust-e);

- Building community based self-regulation systems (e.g. e-Engineering Hub); and

- Providing online dispute solutions (e.g. Cybersettle, WebMediate).

- Many technologies are also adopted to foster trust building in e-Business by improving online security and privacy (e.g. confidentiality of sensitive information, integrity of critical information, prevention of unauthorized copying or use of critical information, traceability of digital objects, management of risks to critical information, and authentication of payment information), which include:

- Identity : Public key infrastructure (PKI) based certificate authorities are commonly used to underpin digital identities. Companies (e.g. Verisign, ValiCert) provide mechanisms and services to outsource validation and verification.

- Digital Credentials : Emerging privilege management infrastructures (PMIs) address the very basic need of certifying attributes associated to users and enterprises such as credit card numbers, certified credit limits, ranking information, etc. 
- Anonymity: Anonymity services are becoming important on the Internet to protect customers' privacy and security. Companies (e.g. Zero-Knowledge Systems) offer an online anonymity service based on encryption mechanisms and IP masking techniques.

- Guaranteed message delivery: Technologies are adopted to assure secured message exchanges between business partners. For example, EDI and WebEDI messaging and business interaction infrastructures provide reliable and trusted infrastructures underpinning interactions.

- Notarisation: Notarisation services provide evidence of the existence of documents and messages at particular points in time. Surety enables users to notarise electronic files, guaranteeing the content and verifying it for a long period. Timestamp.com provides a service to digitally timestamp documents.

- Storage: The storage of critical digital documents is extremely important, especially for long periods of time, as it is the foundation of accountability. Infrastructure technologies (e.g. Storage Area Networks and Network Attached Storage) provide high availability, replication and survivability of stored documents.

Despite the considerable efforts in both business and technology aspects, lack of trust is still a major barrier for the development of e-Business, particularly for SMEs due to the shortage of resources and low creditability (e-Hub Technical Annex, 2001). Much research still needs to be performed to improve trust building in eBusiness, particularly in the complex B2B domain. For example, researchers (e.g. Andersson and Sörvik, URL2; Ong, URL3; EC, 1997) point out the importance of building transparency and predictability e-Business platforms to trust building. Another more important area is the integration of trust building services with eBusiness processes. These are particularly important for the complex e-Engineering environments. It is desired that e-Engineering platforms facilitate the trust building between ESPs and their Clients through the engineering services it provides.

Trust building is one of the major concerns for the development of the e-Hub project. Particular attention is paid to how the e-Hub could facilitate SMEs to build and strengthen the (initial) trust with their potential client(s). This project not only adopts the existing business and technology approaches (e.g. self-regulation, legal conditions, TTP, data access right, time stamps, PKI, etc.), but also emphasizes building trust through collaborative PP conducted on the e-Hub, which offers a transparent, predictable and traceable collaborative workspace.

\section{THE E-ENGINEERING HUB}

The aim of the e-HUBs project was to develop a universal collaborative engineering platform which offers a system-based approaches to e-engineering trust building, enterprise modelling and process sharing, SME services hosting and re-engineering of collaborative workflows. There are five business steps in the e-Hub facilitated collaboration process: 1) deciding what to partner; 2) finding the right partner; 3) agreeing with that partner what, how, when to do it; 4) executing the plan developed in stage 3; and 5) dissolving the partnership. The e-Hub is based on the observation that stage 3 is underdeveloped, under recognised and hence the biggest obstacle between potential partnering and actual partnering. The central concept of the e-Hub is mainly to address the problem raised at this stage. 


\subsection{The Role of the e-Hub}

As illustrated in Figure 1, the e-Hub, through its value added services, provides some dedicated e-Engineering brokerage services for distant co-engineering. In general, there are three kinds of services:

- A Basic Collaboration Platform (BCP), which offers various basic collaborative services such as email, fax, diary, calendar, online chatting room, secured messaging exchange, secured data storage, authority and access right control, language translation, etc.

- A collaborative project preparation, planning and contract negotiation platform, which is the core of the e-Hub. Essentially, the e-Hub workspace is a generic negotiation platform allowing users to define, plan and negotiate various engineering and contractual issues during the project preparation stage.

A group of human specialists/organisations which form the e-Hub community. The community is responsible for verifying the qualifications of the companies that register to it and could provide endorsement to the registered members according to their performance and ratings.

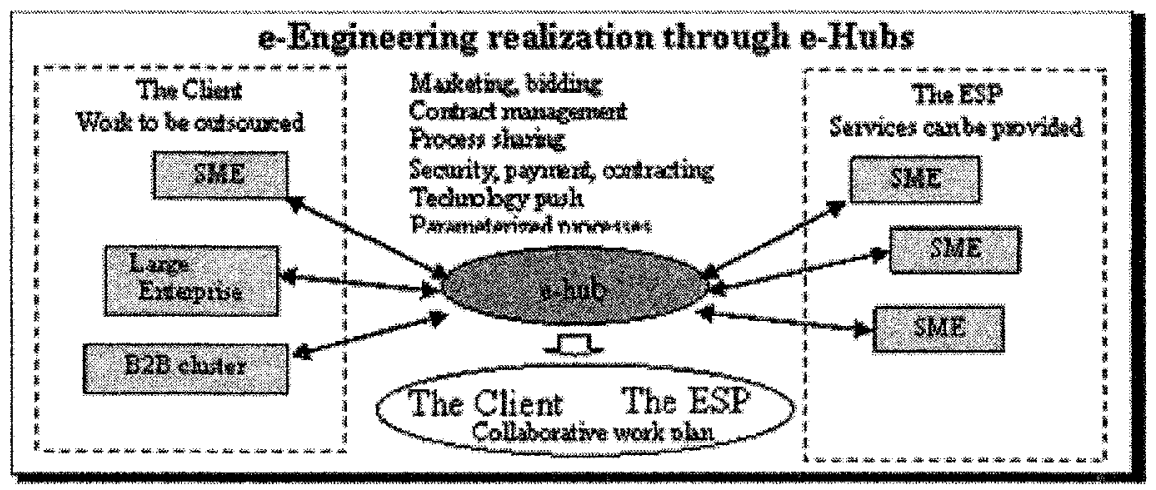

Figure 1. The Collaborative/Intermediating Role of the e-Hub

This study focuses on how the e-Hub's functional architecture could foster the trust building between ESPs and clients. Advanced information technologies and Webhosted collaborative engineering form the baseline of the core of the e-Hub. On top of that, incremental layers of additional services are built. Each service system offers dedicated e-Engineering functions at increasing subsystem scales. The e-Hub is configured by offering transparent collaboration templates to each of these systems. Its services are defined and developed for process sharing and configuration based on the process templates for effective collaboration.

\subsection{The e-Hub's Functional Architecture}

Collaborative PP is viewed by the e-Hub as a managed process that transparently generates a set of comprehensive planning documents. They may contain both 
structured models and unstructured documents. The added value of the e-Hub is that the generation process is collaborative in nature and logically ordered, driven by structured content exchange. Both aspects are embodied in a formal Project Planning Model (PPM), which is a collection of models. Each of these models consists of a PP process model, representing with workflow (WF) models that incorporates the coordination logic of how the project partners negotiate and reach a resolution on one of the aspects that need to be agreed. Each of the WF models operates on one or more content templates, which controls who has read or written access to which field (Figure 2).

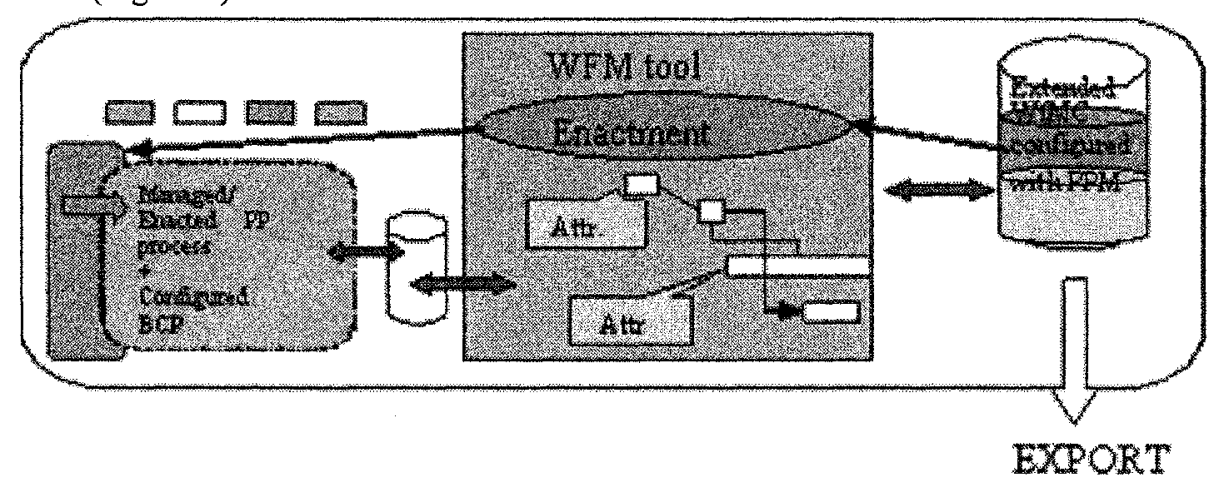

Figure 2. The conceptual model of the e-Hub's functional architecture

The key features could be summarised as follows (Figure 3):

- To facilitate project definition and planning, a meta workflow, developed based on the generic PP process, is embedded in the e-Hub workspace as a generic PP process template. This workflow will guide users going through every key project definition and planning stage such as briefing project charter, clarifying scope statement, and defining execution plan. Various essential PP issues are covered in the execution plan such as project schedule, quality plan, risk plan, communication protocol, and resource plan, with each guided by a sub-workflow.

- Meanwhile, a generic negotiation workflow is also embedded in the e-Hub platform. The enactment of this workflow guides users through the general negotiation process (e.g. propose, check, modify, re-check, re-modify, etc.).

- Besides the generic PP process workflow, various attribute templates for each of the PP issues have been developed and embedded in the e-Hub. These templates, built based on both theoretical studies (e.g. PMBOK \& Process Protocol) and industrial scenarios (D5.1, 2003), specify all the key elements which every project plan should address. The negotiation between the Client and the ESP regarding each project plan is basically to address these attributes specified in the templates.

- To facilitate contract negotiation, the e-Hub contains various engineering service contract templates covering different engineering areas and situations. Users can negotiate and specify the details of the contract and legal issues through the e-Hub's Online Contracting System (OCS). The collaborative work plans generated through the e-Hub platform will be integrated into the OCS and form the core of the engineering service contract. Moreover, the e-Hub also provides a contracting workflow which 
highlights the key contractual issues for each particular engineering service outsourcing situation so that users can finalise all the key issues before they sign the contract.

- On a technical level, the e-Hub provides some unique tools facilitating collaborative PP such as PP whiteboard and annotation functions. The annotation function allows users to mark all the changes made in proposals and the reasons of changes during the negotiation process. This information will be stored in the history folder for future checking. This also contributes to the effort of making e-Hub a transparent and traceable environment for joint work plan definition and negotiation.

-

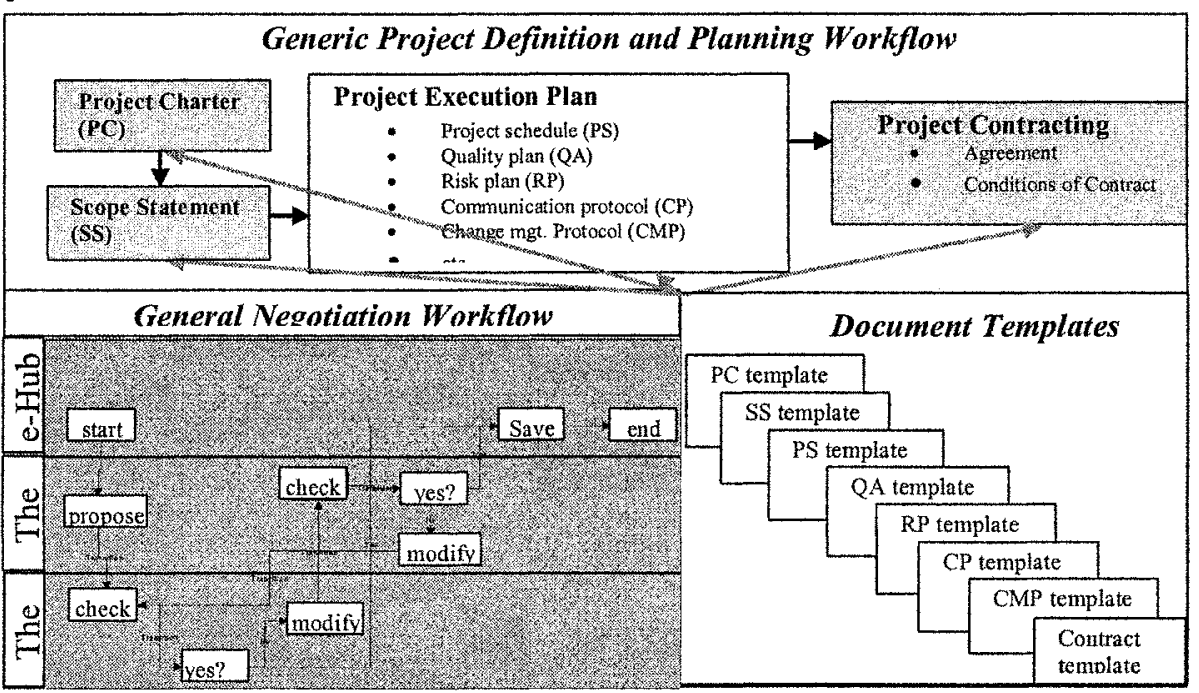

Figure 3. Generic project definition and planning workflow embedded in the e-Hub

\section{DISCUSSION}

The e-Hub's functional architecture provides a unique approach to fostering trust building between clients and ESPs. Some of the key advantages can be summarised as follows:

- First of all, the collaborative project definition and planning process conducted on the e-Hub workspace provides an opportunity for both parties to further strengthen the initial trust built through other approaches (e.g. company profiles and certificate from TTP). Although it might not be necessary for a $\mathrm{B} 2 \mathrm{C}$ environment, the collaborative $P P$ is particularly important for the engineering outsourcing environment, which, as the first step of collaboration, evidences both party's capabilities, goodwill and integrity. This cannot be achieved through any existing initial trust building approaches/services.

- Secondly, the collaborative PP is guided by a generic PP workflow and related attribute templates, which are specific to each particular engineering outsourcing situation. Such an approach is open, transparent, traceable and predictable to all the project planning participants. For those SMEs who are not familiar with the project 
planning process, this approach will help them to develop the project plan, and further build the Client's trust to the ESP. Meanwhile, the negotiation parties are not passive during the PP process, they can suggest, modify and improve the project plan attributes during the negotiation process. This leaves them enough flexibility to conduct the PP. A collaborative project plan developed based on such an approach is helpful to build confidence for project execution.

- Thirdly, the particular technologies adopted in the e-Hub platform such as the annotation function and PP whiteboard improve the transparency, traceability, and predictability of the e-Hub workspace. For example, changes made by any party will be marked with ownership, reasons/suggestions for change and time highlighted with different colours. Furthermore, the generated project plan will be exported to other systems (e.g. e-room or other WFM systems), which will be used to monitor the overall project execution process.

- Finally, the contract negotiation conducted on the e-Hub platform also contributes to the trust building between clients and ESPs. Some of the major advantages include: 1) the contract signed based on the collaborative project plan is firm and solid;2) the contract template and the contract negotiation process templates provide a transparent contract negotiation background. This avoids the problem caused by using any party's existing contract templates. A relatively fair contract is the basis for trust building.

The seismic engineering testbed demonstrates how the e-Hub could facilitate the trust building between the Client and the ESP through both general trust building services and the collaborative PP facilitated trust building approach. This testbed has been evaluated through both expert evaluation clinic and online demonstrations. The results (D6.3) show that the experts from both academia and industry are very positive about the trust building approach though some of the technical details need to be further improved. It is particularly pointed out that building services should not only be limited to current business and technology approaches, engineering service based trust building models should be developed to foster trust building in an eEngineering environment.

\section{CONCLUSION}

This paper presented the engineering e-Hub's functional architecture and discussed its particular role in fostering trust building for SMEs in engineering outsourcing environments. Unlike general e-Markets, where trust services focus on building a social trust environment (e.g. legal systems and community self-regulation) and adopting latest technologies (e.g. security and privacy protection approaches), this study emphasizes the importance of collaborative project planning as an approach of trust building for SMEs involved engineering outsourcing service. The emphasis is the seamless integration of trust building services into the e-Business/ e-Engineering process.

The engineering e-Hub, through a particularly designed functional architecture, provides an open, transparent, traceable and predicable workspace for project participants to conduct collaborative project planning. Guided by the generic PP workflow and related attribute templates, project participants are able to conduct collaborative project planning systematically. This is particularly important for 
complex e-Business situations like engineering outsourcing with the involvement of SMEs. Partners could demonstrate their expertise, resources, willingness of cooperation and integrity during the collaborative PP process. This will strengthen the initial trust gained through other trust services. Furthermore, the e-Hub online contracting system not only helps users to focus on the key contract issues, but also provides a fair basis for contract negotiation. The contract signed based on the collaborative project plan paves the way for the smooth transaction of the project.

Although the concept of the e-Engineering Hub and the particular trust building approach developed in this project are still immature, it has demonstrated the great potential for the enhancement of trust building in B2B environment. The key concept has been adopted in the EU-funded SEEMseed project (Study, Evaluate, and Explore in the Domain of the Single Electronic European Market, IST-1502515-STP).

\section{Acknowledgements}

The e-Hubs project is supported by the European Commission under the IST programme (Contract no: IST-2001-34031). The authors would like to acknowledge the financial support of the European Commission, and record their appreciation to the e-Hubs project partners for their contributions to the project.

\section{REFERENCES}

Andersson, T. and Sorvik, J. Enabling Trust in the Digital World. URL2: http:/www.iked.org/pdf/GlobalTrustCenter.pdf, accessed 25/01/05.

Baessler, E., Mejía, R., Grop, A. V., Crehan, P., and Ren, Z., 2004. D6.3: Evaluation Approach and Benchmark Results. e-HUBs Project Deliverable. IST project: (IST-2001-34031).

Blau, P. (1964). Exchange in Power and Social Life. John Wiley, New York.

Blomqvist, K. and Ståhle, P. Trust in Technology Partnerships. URL5: htp://www stahle, fi/material/Trust in technology partnerships.pdf, accessed 25/01/05.

e-HUBs Project Technical Annex-1 (2001) e-Engineering enabled by Holonomic and Universal Broker Services, Description of Work. IST project: (IST-2001-34031).

Elo, M. (2003). National Culture and Its Impact on Trust and Cooperation in International Business Networks: Some Empirical Evidence from a Greek-Finnish Business Network. URL1: http:/www.impgroup org/uploads/papers/4328.pdf, accessed 25/01/05.

European Commission (1997). Fifth Annual Report of the European Observatory for SMEs. Brussels.

Mayer, R.C. Davis, J.H. \& Schoorman, F.D. (1995). An Integrative Model of Organizational Trust, Academy of Management Review, 20(3), 709-734.

McKnight, D.H., and Chervany, N.L. (1996). The Meanings of Trust, University of Minnesota MIS Research Center Working Paper Series, WP96-04, Minneapolis.

McKnight, D.H, and Chervany, N.L. (2001). Conceptualizing Trust: A Typology and E-Commerce Customer Relationships Model, In Proceedings of the $34^{\text {th }}$ Annual Hawaii International Conference on Systems Science, Maui, Hawaii, IEEE Computer Society Press.

Ong, C.E. B2C E-Commerce Trust in Redress Mechanism (Cross Border Issues), URL3: http:/proceedings.informingscience.org/LS2003Proceedings/docs/018Eang.pdf, accessed 25/01/05.

Project Management Institute, (2000). A Guide to the Project Management Body of Knowledge (PMBOK). Project Management Institute Inc. Newtown Square, PA.

Ren Z., Anumba C.J., Hassan, T.M., (2003). D5.1: Basic Collaborative Engineering Web Services and Functional Architecture to Embed in the e-Hubs. e-HUBs Project Deliverable. IST project: (IST2001-34031).

Ren, Z., Anumba, C.J., Hassan, T.M., (2004). D5.2: Set of Working e-Engineering Services dedicated for Seismic Engineering Demonstrator. e-HUBs Project Deliverable. IST project: (IST-2001-3403I).

Schumacher, C. The Influence of Trust on SMEs Co-Operation Structuring and Performance. URL4: hitp//nzae org.nz/files/\%239-SCHUMACHER.PDF, accessed 25/01/05. 